\title{
CRYSTALLIZATION, PRELIMINARY X-RAY ANALYSIS AND PATTERSON SEARCH OF A NEW ASPARTIC PROTEASE ISOLATED FROM HUMAN URINE
}

\section{Fernanda Canduri ${ }^{1}$, Livia G.V.L.Teodoro ${ }^{2}$, Carla C.B. Lorenzi ${ }^{1}$, Roseli A.S. Gomes ${ }^{2}$, Marcos}

R.M. Fontes ${ }^{3}$, Raghuvir K. Arni ${ }^{1}$ and Walter F. de Azevedo Jr. ${ }^{1 *}$

${ }^{1}$ Departamento de Física, Instituto de Biociências, Letras e Ciências Exatas, UNESP, São José do Rio Preto, SP 15054-000; ${ }^{2}$ Departamento de Bioquímica Celular e Biofísica, Faculdade de Medicina do Triângulo Mineiro, FMTM Uberaba, MG 38015-050, Brazil; ${ }^{3}$ Departamento de Física e Biofisica, IB, UNESP, Botucatu, SP.

Received April 29, 1998

Received after revision, June 22, 1998

\begin{abstract}
Aspartic protease (EC 3.4.23) make up a widely distributed class of enzymes in animals, plants, microbes and, viruses. In animals these enzymes perform diverse functions, which range from digestion of food proteins to very specific regulatory roles. In contrast the information about the well-characterized aspartic proteases, very little is known about the corresponding enzyme in urine. A new aspartic protease isolated from human urine has been crystallized and $\mathrm{X}$-ray diffraction data collected to $2.45 \AA$ resolution using a synchrotron radiation source. Crystals belong to the space group $P 2_{1} 2_{1} 2_{1}$. The cell parameters obtained were $\mathrm{a}=50.99, \mathrm{~b}=75.56$ and $\mathrm{c}=89.90 \AA$. Preliminary analysis revealed the presence of one molecule in the asymmetric unit. The structure was determined using the molecular replacement technique and is currently being refined using simulated annealing and conjugate gradient protocols.
\end{abstract}

Key words: Aspartic protease, crystallography, drug design, synchrotron, X-ray analysis.

\section{INTRODUCTION}

Aspartic proteases are a group of proteolytic enzymes of the pepsin family that share the same catalytic mechanism and usually function in acid solution (1). These proteins are classified as the fourth major class of proteolytic enzymes, distinct from the serine, cysteine, and metalloproteases (2).

They are broadly distributed in nature from retroviruses to humans. The functions of the enzymes are manifold from the nonspecific digestion of proteins to the highly specialized processing of proteinaceous substrates. The substrate-binding sites of aspartic proteases, being extended, are capable of interacting with up to seven amino acid residues of a substrate (3). Acid proteases are so called because they have an optimal catalytic activity at acid pH. They include mammalian digestive enzymes such as pepsin and cathepsin $D$, microbial proteases such as those

\footnotetext{
* To whom correspondence should be addressed
} 
produced by Penicillium janthinellum (penicillopepsin) (4,5), Rhizopus chinensis (rhizopuspepsin) (6), and Endothia parasitica (endothiapepsin) (6) and probably certain enzymes involved in mammalian control processes, for example renin, which acts on angiotensinogen (7). The acid proteases are characterized by the presence of two aspartic acid residues at the active site and most are inhibited by the microbial peptide analogue, pepstatin. They tend to cleave between hydrophobic amino acids but secondary interactions are important in the definition of their specificity (8). Extensive sequence homologies have been observed among the enzymes belonging to this family. Tertiary structures have been determined by $\mathrm{X}$-ray crystallography for three microbial aspartic proteases, namely penicillopepsin (9), Endothia parasitica proteases (10), Rhizopus chinensis proteases (11), and the mammalian homologue, pig pepsin (12). All have broadly similar structures and this, together with the sequence homologies, would appear to indicate that all members of the family are likely to have similar three-dimensional structures. Therefore all should operate through a common catalytic mechanism for the hydrolysis of the substrates. However, substantial differences in substrate specificity, optimum $\mathrm{pH}$ and stability have been shown to exist among the various members of the family (13).

This communication describes the crystallization, preliminary $\mathrm{X}$-ray analysis and Patterson search of an aspartic protease extracted from human urine. The investigation was made in order to gain further insight into the chemistry and functions of this protein.

\section{MATERIAL AND METHODS}

Purification and Crystallization: The aspartic protease has been extracted from urine of healthy individuals using ion exchange chromatography (DEAE and Mono $Q$ ) and gel filtration (Superdex 10/75). It has a molecular weight of approximately $35 \mathrm{kDa}$, determined from sizeexclusion chromatography and electrophoresis gel (SDS-PAGE). This aspartic protease has a single chain, two domains and maximum proteolytic activity in acidic $\mathrm{pH}$ values, as observed for other members of this family.

Lyophilized urinary aspartic protease was dissolved in deionized water (15 $\left.\mathrm{mg} \mathrm{ml}^{-1}\right)$ and centrifuged for $10 \mathrm{~min}$ at $18,000 \mathrm{rpm}$ at $25^{\circ} \mathrm{C}$. The clear supernatant was mixed $1: 1(\mathrm{v} / \mathrm{v})$ with the reservoir solution that contained the appropriate buffer and precipitant. Crystals of urinary aspartic protease have been obtained in several different crystallization conditions, using the hanging drop vapor diffusion and sparse matrix methods (14). The best crystals were obtained after 1 week growth from drops in which $3 \mu \mathrm{l}$ of enzyme solution was mixed with equal volume of $0.1 \mathrm{M}$ Hepes buffer ( $\mathrm{pH} 7.0$ ) containing $2 \%$ polyethylene glycol 400 and $2.0 \mathrm{M}$ ammonium sulfate. Crystal were mounted in capillary tubes of borosilicate glass for X-ray data collection.

$X$-ray data collection and processing: X-ray diffraction data were firstly collected from a single urinary aspartic protease crystal at room temperature using a R-AXIS IV imaging plate system and graphite monochromated $\mathrm{Cu} \mathrm{K} \alpha \mathrm{X}$-rays radiation generated by a Rigaku RU300 rotating 
anode generator operated at $50 \mathrm{kV}$ and $100 \mathrm{~mA}$ at a crystal to detector distance of $150 \mathrm{~mm} .40$ frames were collected using a oscillation range of $2.5^{\circ}$. The exposure time per frame was 20 $\min$. The X-ray diffraction data were processed to $2.8 \AA$ resolution and scaled using the program PROCESS (15). A second X-ray diffraction data set were collect at a wavelength of $1.38 \AA$ using the Synchrotron Radiation Source (Station PCr, Laboratório Nacional de Luz Síncrotron, LNLS, Campinas, Brazil) and a $30 \mathrm{~cm}$ MAR imaging plate detector (MAR Research) with an exposure time of $3 \mathrm{~min}$ per image at a crystal to detector distance of $175 \mathrm{~mm}$. Using an oscillation range of $1.5^{\circ}, 65$ images were collected and the raw X-ray diffraction data were processed to $2.45 \AA$ resolution using the program DENZO (16) and scaled by the program SCALEPACK (16). Auto indexing procedures, combined with analysis of the $\mathrm{X}$-ray diffraction pattern and averaging of equivalent intensities was used in the characterization of the Laue symmetry.

Molecular replacement: The X-ray data collected using synchrotron radiation were used in the molecular replacement. The crystal structure of the human urinary aspartic protease was determined by standard molecular replacement methods using the programs AMoRe (17) and XPLOR (18). The atomic coordinates of ten different aspartic proteases deposited in the PDB (Brookhaven Protein Data Bank) were used as search models. All solvent and ligant molecules were removed from the search models and the temperature factors for all atoms were set to 20.00 $\AA^{2}$. The search models which presented more than one subunit were modified, so that only one subunit was used in the molecular replacement. The atomic coordinates for all search models were translated so that their centre of gravity is at the origin, they were also rotated so that the principal axes of inertia of the search models are parallel to the orthogonal axes. The PDB accession numbers and identification of the search models are listed in Table 1. Initially selfrotation functions based on data in the resolution range 10-4.5 $\AA$ were calculated using a sampling step of $1^{\circ}$ using the program AMoRe (17). Cross-rotation functions were calculated in the resolution range 10-4.5 $\AA$ using a sampling step of $2.5^{\circ}$ for all 10 search models. These calculations were carried out with integration radius of $25 \AA$. The rotation which generated the highest correlation coefficient (CC) was applied to the search models and used in the subsequent translation function computations, based on data in the same resolution range.

The best solutions for each search model were selected based on the magnitude of the $\mathbf{R}_{\text {fador }}$ and correlation coefficient. The search model which generated the best solution were also used for the molecular replacement as implemented in the program X-PLOR (18), using the same resolution range and the same integration radius in order to confirm the solution obtained

Table 1. List of the aspartic protease used as search models for molecular replacement.

\begin{tabular}{cl}
\hline PDB Accession number & \multicolumn{1}{c}{ Protein } \\
\hline leag & Aspartic protease from Candida albicans (Candida pepsin) \\
1lya & Cathepsin D from human liver \\
1 bap & Aspartic protease from Candida albicans \\
$1 \mathrm{mpp}$ & Human renin \\
$2 \mathrm{ren}$ & Pepsin (renin) from Mucor pusillus \\
$4 \mathrm{apr}$ & Renin (recombinant human renin) \\
$1 \mathrm{psn}$ & Aspartic protease from Rhizopus chinensis \\
$3 \mathrm{app}$ & Pepsin 3A from Homo sapiens \\
$1 \mathrm{cms}$ & Penicillopepsin from Penicillium janthinellum \\
\hline
\end{tabular}


using the program AMoRe (17). Translation function for enantiomorphic space groups (P222, $\mathrm{P} 22_{1} 2$ and $\mathrm{P} 22_{1}$ ) has also been computated using the same resolution range and the best search model, in order to confirm the space group.

\section{RESULTS AND DISCUSSION}

The Figure 1 illustrates photomicrograph of urinary aspartic protease crystal suitable for $X$-ray diffraction experiments, with average dimensions of about $0.3 \times 0.4 \times 0.5 \mathrm{~mm}$. The crystal has the orthorhombic space group $\mathrm{P} 2{ }_{1} 2_{1} 2_{1}$ and the volume of the unit cell is $346 \times 10^{3} \AA^{3}$ compatible with one monomer in the asymmetric unit with $V_{m}$ value of $2.17 \AA^{3} / \mathrm{Da}$. Assuming a value of $0.74 \mathrm{~cm}^{3} \mathrm{~g}^{-1}$ for the protein partial specific volume, the calculated solvent content in the crystal is $43.3 \%$ and the calculated crystal density $1.21 \mathrm{~g} \mathrm{~cm}^{-3}$. The X-ray diffraction statistics for the two data sets are summarized in Table 2. Detailed data collection statistics for the Synchrotron data are given in Table 3.

Peak analysis of the self-rotation function did not reveal the presence of any significant local symmetry axis suggesting that a single subunit is contained in the asymmetric unit. This was in agreement with the estimated values of the crystal solvent content and $V_{m}$ value (19).

The results of the molecular replacement using the 10 different search models are listed in Table 4. The correlation coefficients after translation function computation range from 14.4 to $67.8 \%$ and the $\mathrm{R}_{\text {factor }} \mathrm{S}$ range from 35.0 to $54.1 \%$. The search model which presented the best correlation coefficient and $\mathrm{R}_{\text {factor }}$ was the pepsin $3 \mathrm{~A}$ from Homo sapiens (Accession number: $1 \mathrm{psn})$. This search model was also submitted to molecular replacement using the program $\mathrm{X}$ PLOR, and the solution obtained after the translation search was $\left(\alpha=23.6^{\circ}, \beta=47.0^{\circ}, \gamma=59.8^{\circ}\right.$, $x=0.314, y=0.029, z=0.443, R_{\text {factor }}=34.6 \%$ ) close to the one obtained by AMoRe (17)

Translation functions for enantiomorphic space groups (P222, P222 $\left.1, \mathrm{P} 2{ }_{1} 2_{1} 2\right)$ has been computated using the coordinates of pepsin $3 \mathrm{~A}$ as search model and the results are listed in Table 5. The correlation coefficients after translation function computation for the 3 enantiomorphic space groups range from 26.4 to $38.0 \%$ and the $\mathrm{R}_{\text {fador }} \mathrm{S}$ range from 46.4 to $51.1 \%$, which strongly indicates that the correct space group is $\mathrm{P} 22_{1} 2_{1} 2_{1}$.

The urinary aspartic protease is inhibited by the microbial peptide analogue, pepstatin. In order to obtain the structure of the binary complex between the urinary aspartic protease and pepstatin co-crystallization experiments have been initiated

The origin of the urinary aspartic protease still unclear. The analysis of the molecular replacement solution indicates that the urinary aspartic protease structure shows high similarity with the structure of the pepsin. The partially refined model of the urinary aspartic protease is 


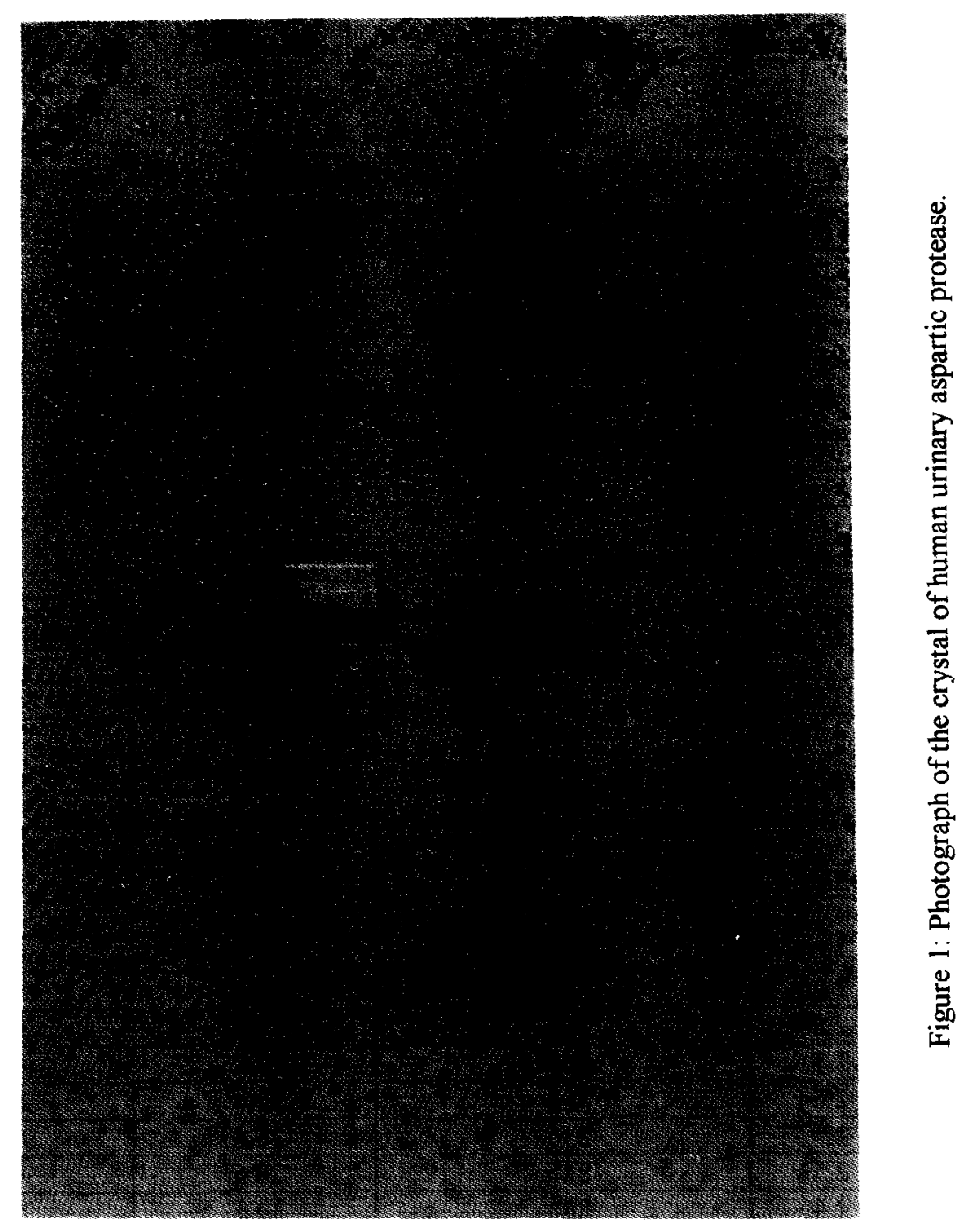


Table 2. Data collection statistics for both data sets.

\begin{tabular}{lcc} 
& R-AXIS IV & LNLS \\
\hline $\mathrm{a}(\AA)$ & 51.08 & 50.99 \\
$\mathrm{~b}(\AA)$ & 75.91 & 75.56 \\
$\mathrm{c}(\AA)$ & 89.99 & 89.90 \\
Number of Measurements with I>2 $\sigma(\mathrm{l})$ & 17686 & 24189 \\
Number of Independent Reflections & 10232 & 13134 \\
$\mathrm{R}^{*}{ }_{\text {sym }}(\%)$ & 10.4 & 7.7 \\
Highest Resolution Shell $(\AA)$ & $3.5-2.8$ & $2.51-2.45$ \\
Completeness in the highest Resolution Shell (\%) & 79.3 & 99.1 \\
$\mathrm{R}^{*}$ sym in the Highest Resolution Shell $(\%)$ & 18.5 & 30.3 \\
\hline
\end{tabular}

${ }^{*} \mathrm{R}_{\text {sym }}=100 \sum \Sigma|\mathrm{I}(\mathrm{h})-<\mathrm{I}(\mathrm{h})>| / \Sigma \mathrm{I}(\mathrm{h})$ with $\mathrm{I}(\mathrm{h})$, observed intensity and $<\mathrm{I}(\mathrm{h})>$, mean intensity of reflection h over all measurement of $I(h)$.

Table 3. Detailed data collection statistics for the synchrotron data

\begin{tabular}{|cc|c|c|c|}
\hline Resolution Range & $\begin{array}{c}\text { Number of } \\
\text { independent reflections }\end{array}$ & $\mathbf{R}_{\text {sym }} \mathbf{( \% )}$ & $\begin{array}{c}\text { Completeness } \\
(\%)\end{array}$ \\
\hline 13.00 & 5.86 & 916 & 4.2 & 94.4 \\
\hline 5.86 & 4.73 & 888 & 6.1 & 98.1 \\
\hline 4.73 & 4.15 & 895 & 6.1 & 99.0 \\
\hline 4.15 & 3.78 & 894 & 7.0 & 99.8 \\
\hline 3.78 & 3.52 & 892 & 7.7 & 99.9 \\
\hline 3.52 & 3.31 & 881 & 8.3 & 99.7 \\
\hline 3.31 & 3.15 & 864 & 9.8 & 99.5 \\
\hline 3.15 & 3.02 & 862 & 12.1 & 99.4 \\
\hline 3.02 & 2.90 & 880 & 15.1 & 99.5 \\
\hline 2.90 & 2.80 & 861 & 17.4 & 99.8 \\
\hline 2.80 & 2.71 & 865 & 20.2 & 99.2 \\
\hline 2.71 & 2.64 & 886 & 22.4 & 99.0 \\
\hline 2.64 & 2.57 & 836 & 26.4 & 98.8 \\
\hline 2.57 & 2.51 & 846 & 28.5 & 98.9 \\
\hline 2.51 & 2.45 & 868 & 30.3 & 99.1 \\
\hline All Reflections & 13134 & 7.7 & 98.9 \\
\hline
\end{tabular}


Table 4. Eulerian angles and fractional coordinates after translation function computation.

\begin{tabular}{|l|c|c|c|c|c|c|c|c|}
\hline Protein & $\alpha\left({ }^{\circ}\right)$ & $\beta\left(^{\circ}\right)$ & $\gamma\left({ }^{\circ}\right)$ & $\mathbf{x}$ & $\mathbf{y}$ & $\mathbf{z}$ & $\mathbf{C C}(\%)$ & $\mathbf{R}_{\text {factor }} \mathbf{( \% )}$ \\
\hline leag & 33.83 & 65.23 & 76.65 & 0.2231 & 0.1861 & 0.2065 & 14.4 & 54.1 \\
\hline 1lya & 156.00 & 46.61 & 121.10 & 0.3169 & 0.4727 & 0.0587 & 39.4 & 46.7 \\
\hline lzap & 122.98 & 39.94 & 38.05 & 0.2905 & 0.2007 & 0.3434 & 14.4 & 55.2 \\
\hline lbbs & 152.67 & 50.03 & 121.40 & 0.3250 & 0.4819 & 0.0588 & 26.5 & 50.8 \\
\hline 1mpp & 21.80 & 90.00 & 4.34 & 0.2000 & 0.1747 & 0.0514 & 16.2 & 53.5 \\
\hline 2ren & 30.54 & 40.01 & 235.18 & 0.3283 & 0.0154 & 0.4332 & 33.1 & 48.4 \\
\hline 4apr & 149.04 & 44.86 & 125.18 & 0.3176 & 0.4861 & 0.0618 & 22.1 & 52.5 \\
\hline 1psn & 24.44 & 46.13 & 59.44 & 0.3224 & 0.0247 & 0.4385 & 67.8 & 35.0 \\
\hline 3app & 146.53 & 46.99 & 307.78 & 0.3058 & 0.4739 & 0.0524 & 20.2 & 53.2 \\
\hline 1cms & 152.27 & 45.15 & 122.87 & 0.3209 & 0.4742 & 0.0610 & 39.3 & 48.9 \\
\hline
\end{tabular}

${ }^{*} \mathbf{R}_{\text {factor }}=100 \times \Sigma \mid F_{\text {obs }}-F_{\text {calc }} / \Sigma\left(F_{\text {obs }}\right)$, the sums being taken over all reflections with $F / \sigma(F)>2$ cuttoff.

Table 5: Fractional coordinates after translation function computation for other possible enantiomorphic space groups.

\begin{tabular}{cccccc}
\hline Space Group & $\mathbf{x}$ & $\mathbf{y}$ & $\mathbf{z}$ & $\mathbf{C C}(\%)$ & $\mathbf{R}_{\text {factor }}(\%)$ \\
\cline { 2 - 5 } $\mathrm{P} 2{ }_{1}{ }_{1} 2$ & 0.0748 & 0.1244 & 0.1889 & 38.0 & 46.4 \\
$\mathrm{P} 222_{1}$ & 0.0702 & 0.0254 & 0.3940 & 38.5 & 46.5 \\
$\mathrm{P} 222$ & 0.0080 & 0.0045 & 0.0104 & 26.4 & 51.1 \\
\hline
\end{tabular}

${ }^{*} \mathrm{R}_{\text {factor }}=100 \times \Sigma \mid \mathrm{F}_{\text {obs }}-\mathrm{F}_{\mathrm{calc}} / \Sigma\left(\mathrm{F}_{\mathrm{obs}}\right)$, the sums being taken over all reflections with $\mathrm{F} / \sigma(\mathrm{F})>2$ cuttoff. 

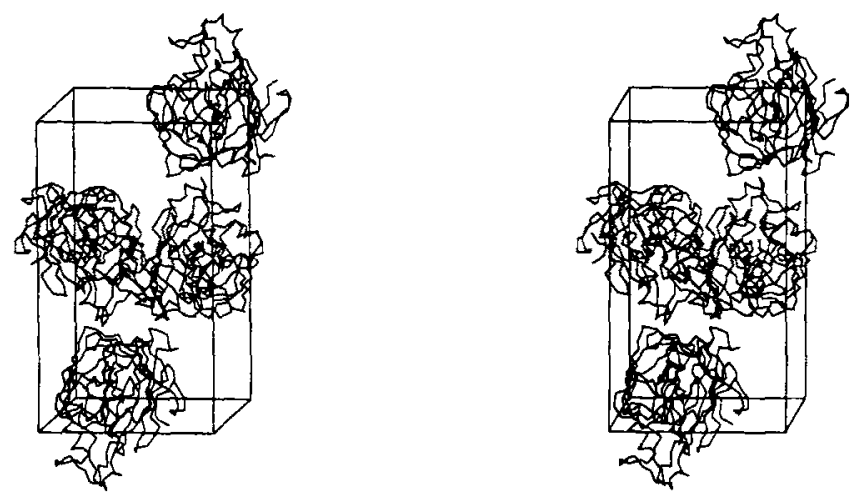

Figure 2: Crystal packing for the partially refined structure of the human urinary aspartic protease.

bilobal, consisting of two predominantly $\beta$-sheet lobes which, as observed in other aspartic protease, are related by pseudo 2-fold axis. Figure 2 presents the crystal packing for the partially refined structure of the urinary aspartic protease. Further refinement using the slow-cooling protocols will be performed using the program X-PLOR (18). The refined model of the urinary aspartic protease will be used for detailed comparison with other proteins of this family.

Acknowledgments: We thank Dr. Paula Kuser and Dr. I. Polikarpov (LNLS) for their help in the synchrotron data collection. This work was supported by grants from FAPESP, CNPq and FUNDUNESP (Brazil).

\section{REFERENCES}

1. Tang, J. \& Wong, R. N. S. (1987). J. Cell Biochem. 33, 53-63.

2. Hara, K., Fukuyama, K., Sakai, H., Yamamoto, K. \& Epstein, W. L. (1993). J. Invest. Dermatol. 100, 4, 394-399.

3. Filippova, I. Yu., Lysogorskaya, E.N., Anisimova, V.V., Suvorov, L.I., Oksenoit, E.S. \& Stepanov, V.M. (1996). Analytical Biochemistry 234, 113-118.

4. Hsu, I-N. (1977). in Acid Proteases, Structure-Function and Biology (ed. Tang, J.) 61 Plenum, New York.

5. Hsu, I-N. Delbaere, L.T.J., James, M.N.G. \& Hofmann, T. (1977). Nature 266, 140-145.

6. Subramanian, E., Swan, I.D.A., Liu, M., Davies, D.R., Jenkins, J.A., Tickle, I.J. \& Blundell, T.L. (1977). Proc. Natl. Acad. Sci. USA 74, 556-559.

7. Tang, J., James, M.N.G., Hsu, I-N., Jenkins, J.A. \& Blundell, T.L. (1978). Nature (London) $271,618-621$.

8. Fruton, J.S. (1976). Adv. Enzym. 44, 1-36.

9. James, M.N.G. \& Sielecki, A. (1983). J. Mol. Biol. 163, 299-361.

10. Pearl, L.H. \& Blundell, T L. (1984). FEBS Lett. 174, 96-101. 
11. Bott, R., Subramanian, E. \& Davies, D.R. (1982). Biochemistry 21, 6956-6962.

12. Andreeva, N.S., Zdanov, A.S., Gustchina, A.E. \& Fedorov, I.A. (1985). in Aspartic Proteinases and their Inhibitors (Kostka, V., ed.), 137-150 Walter de Gruyter, Berlin.

13. Dunn, B.M., Jimenez, M., Parten, B.F., Valler, M.J., Rolph, C.E. \& Kay, J. (1986). Biochem. J. 237, 899-906.

14. Jancarik, J. \& Kim, S. -H. (1991). J. Appl. Cryst. 24, 409-411.

15. Higashi, T. (1990). J. Appl. Cryst. 23, 253-257.

16. Gewirth, D. (1995). The HKL Manual. Yale University, New Haven. Connecticut 06511 USA.

17. Navaza, J. (1994). Acta Cryst. A50, 157-163

18. Brünger, A. T. (1992). X-PLOR Version 3.1: A system for crystallography and NMR. Yale University Press, New Haven.

19. Mathews, B.W. (1968). J. Mol. Biol. 33, 491-497. 